\title{
Articular cartilage defects of the knee: correlation between magnetic resonance imaging and gross pathology
}

Robert L Karvonen, William G Negendank, Susan M Fraser, Maureen D Mayes, Teisa An, Felix Fernandez-Madrid

\begin{abstract}
Magnetic resonance imaging (MRI) of the knee articular cartilage is possible owing to the contrast provided by different signal intensities of adjacent menisci and subchondral bone. The objective of this study was to determine the accuracy of MRI in quantitatively detecting thinning and focal defects of articular cartilage in vivo. High resolution MRI was performed followed by dissection of the knee within one hour of amputations above the knee of eight patients (62-89 years) with peripheral vascular disease. Articular cartilage was examined for erosions, surface irregularities, and appearance. Mean thicknesses of femoral and tibial articular cartilage sagittal sections from MRI were statistically indistinguishable from matched gross thicknesses. In those joints in which cartilage erosions, thinning, or irregularities were detected by MRI the same defects were apparent by gross examination. Cartilage that appeared normal by MRI had a normal gross appearance by gross examination. Thus high resolution MRI can accurately predict gross articular cartilage appearance and thickness, allowing an objective, quantitative, noninvasive assessment of eroded cartilage.
\end{abstract}

Conventional radiographs are not helpful in evaluating cartilage erosions. Magnetic resonance imaging (MRI) is a promising noninvasive means of evaluating joint integrity. Magnetic resonance imaging of the knee defines articular cartilage clearly owing to the contrast provided by different signal intensities of adjacent menisci and subchondral bone. Its usefulness in depicting the normal anatomical landmarks of the knee as well as changes in menisci, ligaments, and periarticular structures has been reported. ${ }^{1-8}$ In addition, Stoller $e t$ al found an excellent correlation between abnormal meniscal signals in MRI and the extent of mucinous degeneration in histopathology of knees obtained from necropsy or after amputation. ${ }^{9}$ Little work has been done to ascertain the value of MRI in assessing discrete articular cartilage defects, though its potential has been recognised in studies of the knee and wrist. ${ }^{2} 10$ 11 Intra-articular injection of contrast agents can help in the detection of cartilage defects, ${ }^{12}$ but its invasive nature and the need to repeat this procedure to assess the progression of erosions make this approach less desirable. Wojtys et al have reported that MRI can detect articular cartilage defects disclosed by arthrotomic or arthroscopic examination. ${ }^{13}$ The pur- pose of this study was to determine whether MRI can be used to detect and measure changes in the thickness and focal architecture of articular cartilage. Preliminary results of this study have been reported in abstract form. ${ }^{14}$

\section{Patients and methods}

Table 1 gives details of the patients studied.

Magnetic resonance imaging was performed on extended knee joints obtained within one hour of amputations above the knee in eight patients with peripheral vascular disease. High resolution, T1 weighted, spin echo coronal and sagittal images were obtained with a $256 \times 256$ matrix in a field of view of $24 \mathrm{~cm}(0.95$ $\mathrm{mm} / \mathrm{pixel}$ ) in either a 1.0 tesla (five knees) or a 1.5 tesla (three knees) Siemen's instrument using opposed surface coils in a Helmholtz configuration as the receiver. Repetition time (TR) was 600 or $700 \mathrm{~ms}$ and echo time (TE) was 22 or $17 \mathrm{~ms}$, respectively. Multiple sagittal images $3 \mathrm{~mm}$ thick were obtained with a gap of $1.5 \mathrm{~mm}$-that is, at $4.5 \mathrm{~mm}$ intervals. The frequency encoding axis was perpendicular to the articular cartilage on the tibial plateau. Chemical shift artefact-that is, overlap of signals from the marrow fat-was not sufficient to interfere with visualisation of the tibial or femoral articular cartilage because the thickness of subchondral bone (which has very low signal) separated the fatty marrow from the articular cartilage.

In our experience $\mathrm{T} 1$ weighted images produced the best contrast between subchondral bone, articular cartilage, and the menisci, and therefore only $\mathrm{Tl}$ weighted images were used for the analysis of articular cartilage thicknesses. Window settings were adjusted to optimise the contrast between articular cartilage and subchondral bone or meniscal cartilage. It should be emphasised that window settings optimised for this purpose are not necessarily the best for obtaining a good photographic reproduction, and thus the quality of the original magnetic

Table 1 Patient data

\begin{tabular}{llll}
\hline $\begin{array}{l}\text { Patient } \\
\text { No }\end{array}$ & Age & Sex & Race \\
\hline 1 & 62 & F & W \\
2 & 79 & F & B \\
3 & 89 & F & B \\
4 & 66 & F & W \\
5 & 84 & F & W \\
6 & 75 & M & B \\
7 & 65 & M & W \\
8 & 74 & F & B
\end{tabular}

Mean age was 74 (SD 9.6) years; $F: M=6: 2 ; W: B=4: 4$. 
resonance image was not fully reproducible in photographs.

Imaging of the intact knee took about 15 minutes and was immediately followed by arthrotomy and pathological examination of the cartilage surfaces and menisci. To provide a quantitative comparison four knee joints (patients 5-8) were sectioned and articular cartilage thicknesses measured. Because the joints were sectioned and measured immediately there were no effects due to freezing or drying of the cartilage. None of the joints studied had an abnormal amount of fluid in the joint. The articular cartilage was examined for erosions characteristic of degenerative joint disease and for surface irregularities and thickness. Menisci were examined for surface irregularities and appearance.

The tibial plateaux and femoral condyles of four of the patients (5-8) were cut into several sagittal sections immediately after MRI (fig 1). In the coronal plane the position of each gross sagittal section was measured, as well as its distance from the centre of the knee. Also in the coronal plane the largest bone width across both condyles was measured, and the distance of each sagittal section from both ends of the largest bone width was recorded. Each gross sagittal section was traced on graph paper. For the femoral condyles an arbitrary starting point was noted on each section and its graph paper tracing, near the posterior aspect of the knee. For the tibial cartilage the flatter surface of the plateau allowed the starting point to be placed exactly at the centre of each side of the plateau. An overlay of parallel lines $1 \mathrm{~cm}$ apart was placed on each sagittal section using the arbitrary point to align the first parallel line, and the articular cartilage thickness was determined to within $0.1 \mathrm{~mm}$, where each line intersected the cartilage surface. The gross sagittal sections were then matched to the corresponding MRI sections using the coronal plane gross measurements and the coronal MRI. The tracings of each gross sagittal section were entered into a computer data base and an overlay image of the gross section matching the distance scale of the MRI was generated on plastic. The overlay with parallel lines $1 \mathrm{~cm}$ apart was placed over the sagittal magnetic resonance images of the corresponding slice, positioned for an exact match, and the cartilage thickness determined for each of the points corresponding to the gross measurements. Magnetic resonance imaging and gross measurements were performed
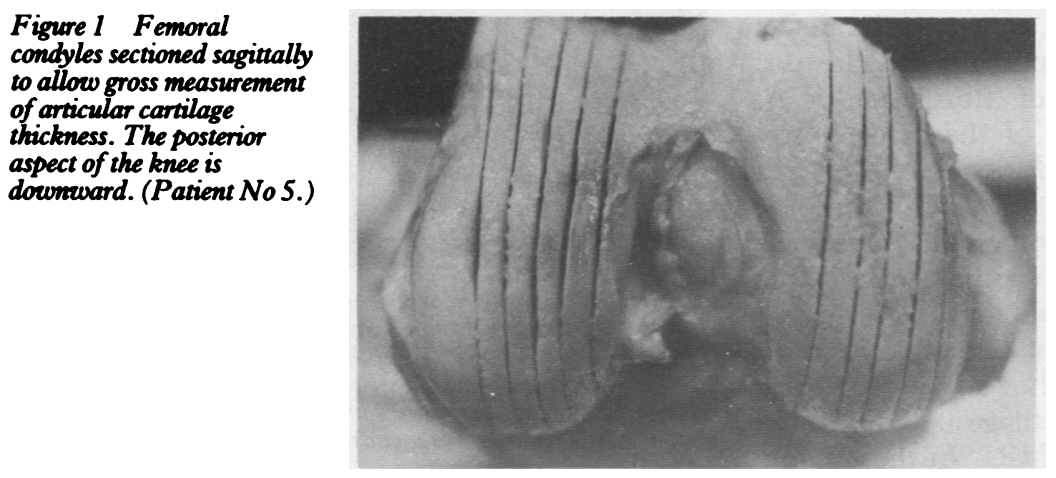

separately at different times to eliminate biased measurements. Magnetic resonance imaging and gross thickness measurements were made with a dissecting microscope and a transparency with parallel lines $0.1 \mathrm{~mm}$ apart, laid over the MR image or the specimen respectively. Measurements in this study were obtained at cartilage sites where partial volume averaging did not occur. Matched cartilage thicknesses were determined for several dozen different articular cartilage sites of each individual knee. Intraobserver variability in measuring articular cartilage thickness for two different observers was $3 \cdot 2 \%$ and $10 \cdot 5 \%$ respectively (table 2 ). Interobserver variability averaged $17 \cdot 8 \%$ (table 3). Although two observers in table 2 and three observers in table 3 were used to test variability, only one observer (with the smallest intraobserver variability, see table 2) was used in all subsequent measurements.

Cartilage and synovial membrane for histology were fixed in $10 \%$ buffered formalin, embedded in paraplast (Fisher Scientific, Livonia, MI), sectioned, and stained with haematoxylin and eosin.

Paired Student's $t$ tests and the standard errors for the differences were used to determine if the matched sets of thickness measurements were different.

\section{Results and discussion}

The articular cartilage specimens showed anatomical changes ranging from normal to severely eroded cartilage with no histological evidence of inflammation. A typical MRI section through normal cartilage shows an even articular surface consistent with the gross examination of the specimen (fig 2). A typical MRI section through a severely eroded femoral condyle shows an articular cartilage surface with obvious abnormalities (fig 3). Figure 4 shows a magnetic resonance sagittal image through a section of thinning articular cartilage flanked by normal regions. Figure 5 shows an example of a small focal defect (about $1 \mathrm{~mm}$ in diameter

Table 2 Intraobserver variability in the measurement of articular cartilage thickness by magnetic resonance imaging

\begin{tabular}{llll}
\hline Observer & $\begin{array}{l}\text { Mean } \\
\text { thickness (SD) } \\
(\text { mm })\end{array}$ & $\begin{array}{l}\text { Coefficient of } \\
\text { variation (\%) }\end{array}$ & $n$ \\
\hline 1 & $1.91(0 \cdot 20)$ & 10.5 & 4 \\
2 & $1.41(0.04)$ & 3.2 & 5 \\
\hline n=number of measurements. & &
\end{tabular}

Table 3 Interobserver variability in the measurement of articular cartilage thickness by magnetic resonance imaging for three observers

\begin{tabular}{lll}
\hline Site & $\begin{array}{l}\text { Mean } \\
\text { thickness }(S D) \\
(\text { mm })\end{array}$ & $\begin{array}{l}\text { Coefficient of } \\
\text { variation }(\%)\end{array}$ \\
\hline 1 & $1.63(0 \cdot 21)$ & 12.8 \\
2 & $1.69(0.50)$ & 29.7 \\
3 & $2.09(0 \cdot 48)$ & 23.2 \\
4 & $1.94(0 \cdot 10)$ & 5.4 \\
Average & & 17.8 \\
\hline
\end{tabular}

Four sites were selected at random. Each observer made one measurement at each site. 
Figure 2 Magnetic articular cartilage with the posterior aspect of the knee to the right $(A)$ and corresponding gross femoral cartilage slice with knee posterior aspect also to the right (B). (Patient No 4.) resonance image of normal
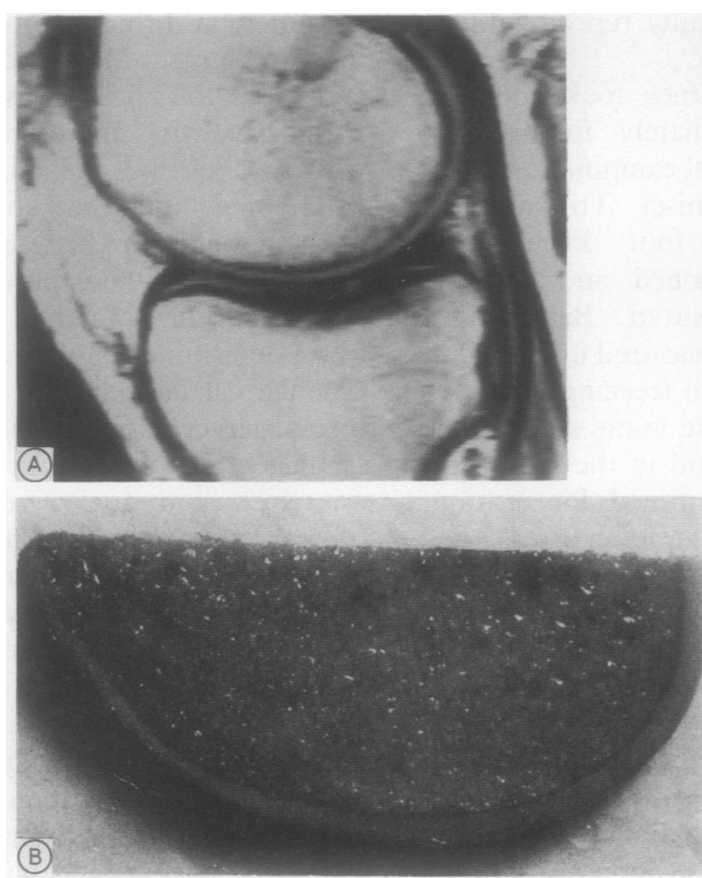

Figure 3 Magnetic resonance image of severely eroded femoral articular cartilage with the posterior aspect of the knee to the right (A) and corresponding cartilage by gross examination with knee posterior aspect downward $(B)$. White arrows on the gross photograph $(B)$ indicate the position of the sagittal magnetic resonance image. (Patient No 8.)
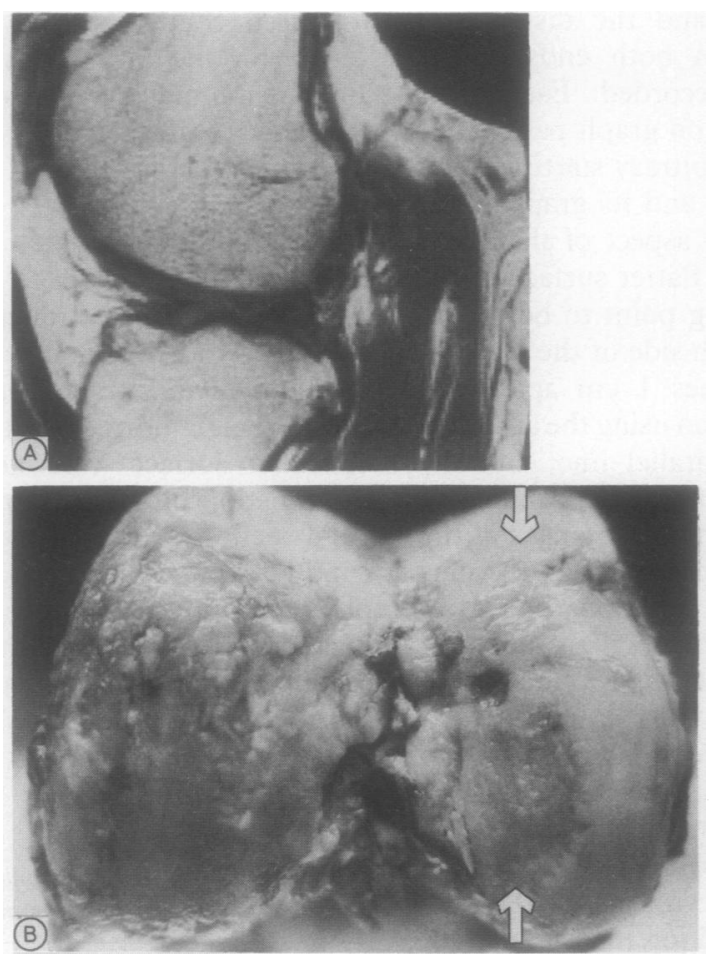

extending down to the bone) detected on a magnetic resonance image and appearing on the corresponding gross photograph. Histological examination of the defect and its surrounding area showed the centre of the defect with an absence of cartilage, many cell clusters, large erosions, and areas of 'fibrillation'. ${ }^{15} \mathrm{At}$ $0.45 \mathrm{~mm}$ from the centre of the defect histological examination showed fraying of the cartilage and fibrillation. At $0.75 \mathrm{~mm}$ from the centre the cartilage was somewhat frayed. At $1.75 \mathrm{~mm}$ and $3.25 \mathrm{~mm}$ from the centre of the defect the cartilage morphology was normal except for a rare cluster of cells at $1.75 \mathrm{~mm}$. Thus histological analysis showed a narrow zone of eroded and abnormal cartilage at and near the defect, in agreement with MRI. In those joints
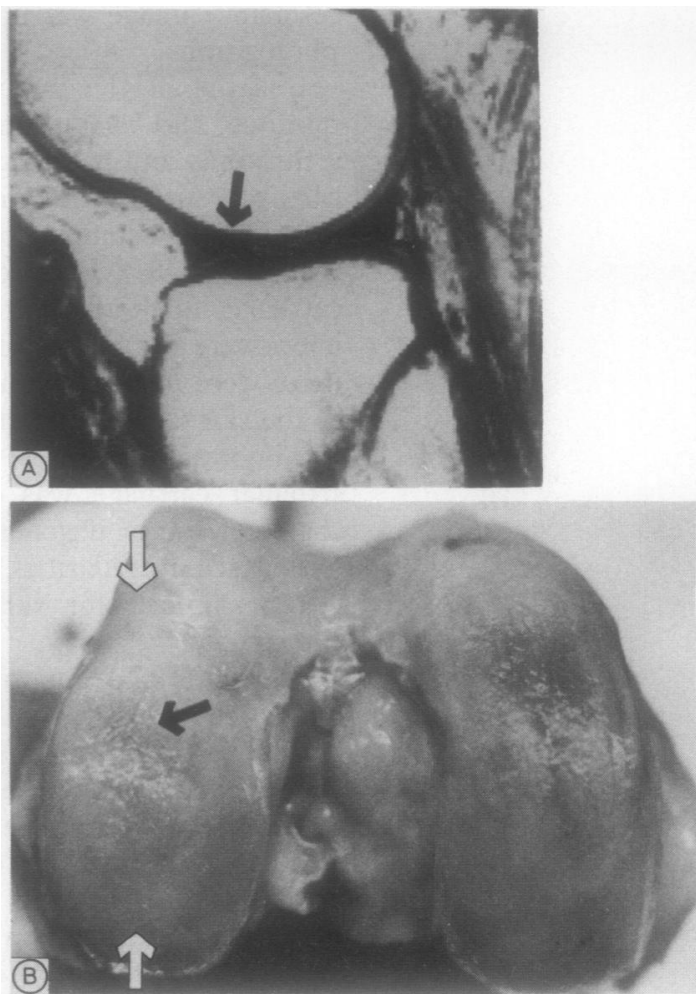

Figure 4 Magnetic resonance image of thinning (arrow) femoral articular cartilage with the posterior aspect of the knee to the right $(A)$ and corresponding thinning (black arrow) by gross examination with knee posterior aspect downward $(B)$. White arrows on the gross photograph $(B)$ indicate the position of the sagittal magnetic resonance image. (Patient No 5.)

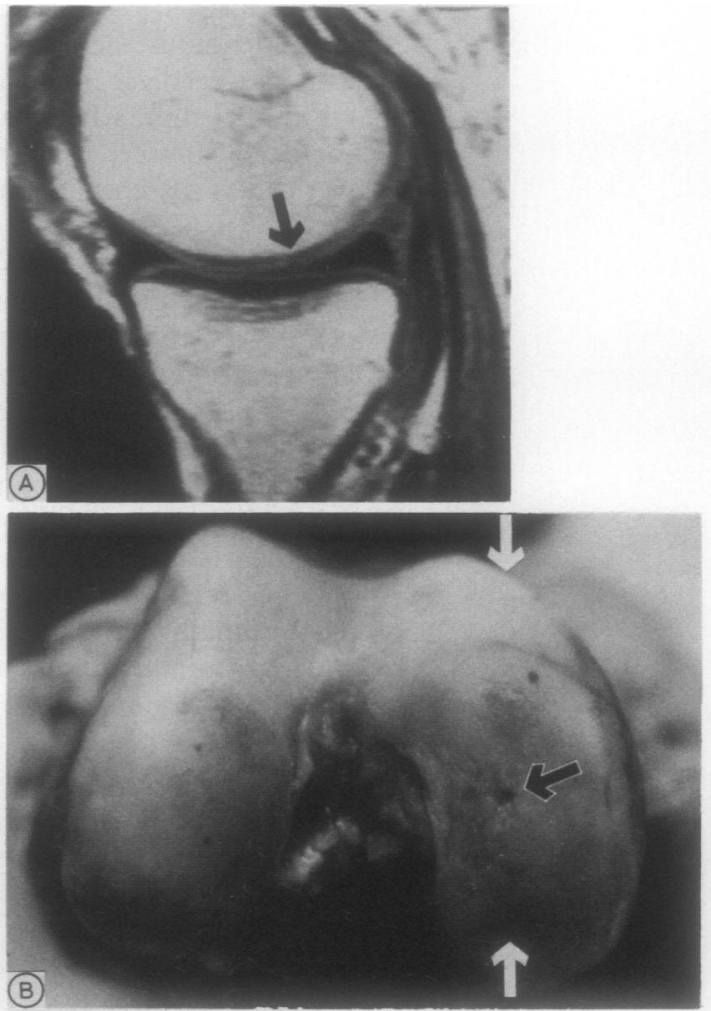

Figure 5. Magneric resonance image of a small defect (arrow) in femoral articular cartilage with the posterior aspect of the knee to the right (A) and the same defect (black arrow) by gross examination with knee posterior aspect downward (B). White arrows on the gross photograph $(B)$ indicate the position of the sagitual magnetic resonance image. (Patient No 3.) 
Table 4 Comparison of human knee articular cartilage thicknesses measured by magnetic resonance imaging $(M R I)$ and gross examination

\begin{tabular}{|c|c|c|c|c|c|c|}
\hline \multirow[t]{2}{*}{$\begin{array}{l}\text { Patient } \\
\text { No }\end{array}$} & \multirow[t]{2}{*}{ Cartilage } & \multirow[t]{2}{*}{ Side } & \multirow[t]{2}{*}{$n$} & \multicolumn{2}{|c|}{$\begin{array}{l}\text { Mean thickness (SD) } \\
\text { in } \mathrm{mm}\end{array}$} & \multirow{2}{*}{$\begin{array}{l}\text { Standard } \\
\text { error } \\
\text { for the } \\
\text { difference }\end{array}$} \\
\hline & & & & $M R I$ & Gross & \\
\hline $\begin{array}{l}5 \\
5 \\
5 \\
5 \\
6 \\
6 \\
6 \\
6 \\
7 \\
7 \\
7 \\
7 \\
8 \\
8 \\
8\end{array}$ & $\begin{array}{l}\text { Femoral } \\
\text { Femoral } \\
\text { Tibial } \\
\text { Tibial } \\
\text { Femoral } \\
\text { Femoral } \\
\text { Tibial } \\
\text { Tibial } \\
\text { Femoral } \\
\text { Femoral } \\
\text { Tibial } \\
\text { Tibial } \\
\text { Femoral } \\
\text { Femoral } \\
\text { Tibial }\end{array}$ & $\begin{array}{l}M \\
\mathrm{~L} \\
\mathrm{M} \\
\mathrm{L} \\
\mathrm{M} \\
\mathrm{L} \\
\mathrm{M} \\
\mathrm{L} \\
\mathrm{M} \\
\mathrm{L} \\
\mathrm{M} \\
\mathrm{L} \\
\mathrm{M} \\
\mathrm{L} \\
\mathrm{L}\end{array}$ & $\begin{array}{r}18 \\
18 \\
13 \\
9 \\
20 \\
17 \\
15 \\
12 \\
20 \\
22 \\
13 \\
12 \\
13 \\
21 \\
8\end{array}$ & $\begin{array}{l}1.3(0.4) \\
1.9(0.5) \\
1.3(0.5) \\
1.6(0.3) \\
2.3(1.0) \\
2.0(0.5) \\
2.6(0.8) \\
1.6(0.4) \\
2.5(0.8) \\
2.2(0.4) \\
2.4(0.9) \\
3.1(1.4) \\
1.5(0.9) \\
2.2(0.8) \\
1.8(0.4)\end{array}$ & $\begin{array}{l}1.3(0.4) \\
1.9(0.5) \\
1.4(0.4) \\
1.7(0.4) \\
2.4(1.2) \\
2.1(0.6) \\
2.6(0.8) \\
1.4(0.5) \\
2.7(0.6) \\
2.2(0.3) \\
2.4(0.7) \\
3.1(1.5) \\
1.5(0.9) \\
2.3(0.8) \\
1.8(0.5)\end{array}$ & $\begin{array}{l}0 \cdot 1 \\
0.2 \\
0.2 \\
0 \cdot 2 \\
0.3 \\
0 \cdot 2 \\
0.3 \\
0 \cdot 2 \\
0 \cdot 2 \\
0 \cdot 1 \\
0.3 \\
0.6 \\
0.4 \\
0.2 \\
0.2\end{array}$ \\
\hline
\end{tabular}

$\mathbf{n}=$ number of different sites of articular cartilage where thickness measurements were performed; $M=$ the medial side; $\mathbf{L}=$ the lateral side.

In every case the paired $t$ test of the means indicated no significant difference between MRI and gross thicknesses ( $>>0.05)$. articular cartilage crossed the section at an angle greater than about $45^{\circ}$ because partial volume averaging $^{16}$ of cartilage signals with those of bone occurred. An example is shown in fig 6 . The cartilage thickness could be measured if the slice through this region were obtained at a different angle where a perpendicular to the articular surface would be parallel to the plane of the MRI section. Partial volume averaging differs from focal areas of denuded bone on the articular surface, in that the latter are usually flanked by cartilage and do not produce a normal signal even if realigned. Tibial articular cartilage thicknesses can be determined over a greater percentage of the surface as the tibial plateau is relatively flat with perpendiculars to more points in its plane parallel to the sagittal sections.

Thus high resolution MRI can accurately predict gross appearance and thickness of articular cartilage, allowing an objective, quantitative, non-invasive in vivo assessment. This non-invasive method may prove valuable in determining the rate of cartilage degeneration and repair, and in assessing the long term effects of drug treatment on eroded articular cartilage.

The authors wish to thank Neil Keller for technical assistance in MRI, Dr George Comondan for generous use of equipment, the Harper Hospital pathology staff for their help in obtaining knee Harper Hospital pathology staff for their help in obtaining knee specimens, and the Michigan Chapter Arthritis Foundation and the Harper-Grace Hospital Radiation Oncology Department for partial support of this project. Magnetic resonance imaging was
performed in the Vaitkevicius MR Center at Harper Hospital. ence between the mean thickness of all th measured points from oross examination and those obtained by MRI (table 4). Thus MR images taken in $3 \mathrm{~mm}$ thick slices can detect variations in cartilage thickness of the order of 1 $\mathbf{m m}$ as well as focal defects of $1 \mathbf{~ m m}$ diameter.

The MRI signals observed in articular cartilage are of higher intensity than those of subchondral bone or menisci. The areas of lower signal intensity were usually thicker under tibial than under femoral cartilage, and in the tibial plateaux were often several millimetres thick, especially in the central region of each side-for example, fig 5 .

The MRI sagittal sections were adjusted to sample signal intensity in a $3 \mathrm{~mm}$ slice thickness. Cartilage thickness could not be obtained in sections where the surface of the femoral

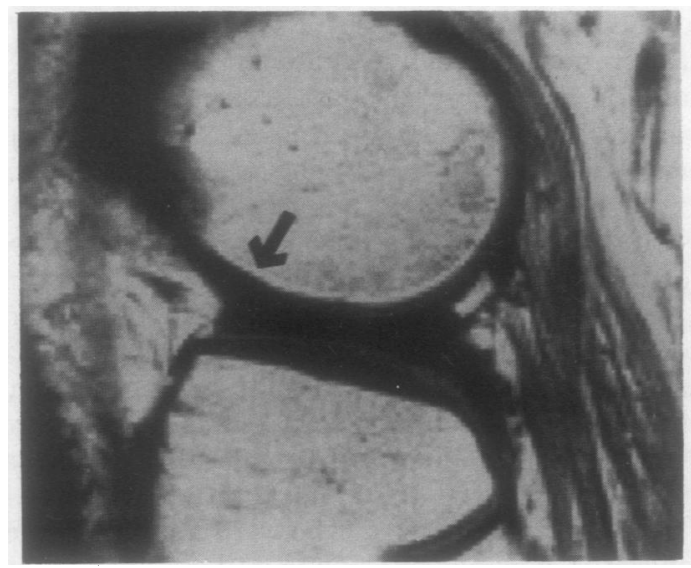

Figure 6 Magnetic resonance image of a femoral articular cartilage region (arrow) showing partial volume averaging of cartilage and bome signals. The posterior aspect of the knee is to the right. (Patient No 7.)
1 Beltran J, Noto A M, Mosure J C, Weiss K L, Zuelzer W, Christoforidis A J. The knee; surface-coil MR imaging at Christoforidis A J. The knee; surface-

2 Burk D L, Kanal E, Brunberg J A, Johnstone G F, Swensen $\mathrm{H} \mathrm{E}$, Wolf G L. 1.5 T surface-coil MRI of the knee. $A \mathcal{F} R$

3 Haggar A $\dot{M}$, Froelich J W, Hearshen D O, Sadasivan K. Meniscal abnormalities of the knee: 3DFT fast-scan GRASS MR imaging. AYR 1988; 150: 1341-4.

4 Jackson D W, Jennings L D, Maywood R M, Berger P E. Magnetic resonance imaging of the knee. Am $\mathcal{F}$ Sports Med 1988; 16: 29-37.

5 Manco L G, Lozman J, Coleman N D, Kavanaugh J H, Bilfield B S, Dougherty J. Noninvasive evaluation of knee Bilfield B S, Dougherty J. Noninvasive evaluation of knee CT. Radiology 1987; 163: 727-30.

6 Mink J H, Levy T, Crues J V. Tears of the anterior cruciate ligament and menisci of the knee: MR imaging evaluation. Radiology 1988; 167: 769-74.

7 Polly D W, Callaghan J J, Sikes R A, McCabe J M, McMahon K, Savory C G. The accuracy of selective magnetic resonance imaging compared with the findings of arthroscopy of the knee. F Bone foint Surg [Am] 1988; 70: arthroscos.

8 Reicher M A, Hartzman S, Bassett L W, Mandelbaum B, Duckwiler G, Gold R H. MR imaging of the knee. Part I. Traumatic disorders. Radiology 1987; 162: 547-51.

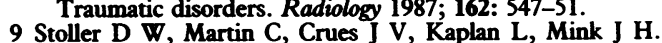
Meniscal tears: pathologic correlation with MR imaging. Meniscal tears: pathologic cort
Radiology 1987; 163: 731-5.

10 Koenig H, Lucas D, Meissner R. The wrist: preliminary report on high-resolution MR imaging. Radiology 1986; 160: 463-7.

11 Li D K B, Adams M E, McConkey J P. Magnetic resonance imaging of the ligaments and menisci of the knee. Radiol

12 Gytys-Morin V M, Hajek P C, Sartoris D J, Resnick D. Articular cartilage defects: Detectability in cadaver knees with MR. AYR 1987; 148: 1153-7.

13 Wojtys E, Wilson M, Buckwalter K, Braunstein E, Martel and intraarticular pathology. Am $\mathcal{f}$ Sports Med 1987; 15: and intragr.

14 Karvonen R L, Negendank W G, Fernandez-Madrid F, Fraser S M. Pathologic correlation of articular cartilage Fraser $\mathbf{S}$ M. Pathologic correlation of articular cartilage defects in MR imaging of the
Rhewe 1988; 31 (suppl): S19.

15 Mankin H J. Normal articular cartilage and the alterations in osteoarthritis. In: Lombardino J G, ed. Nonsteroidal

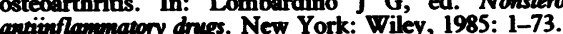

16 Richardson M L, Helms C A. Artifacts, normal variants, and imaging piffalls of musculoskeletal magnetic resonance imaging. Radiol Clin North Am 1986; 24: 145-77. 1986; 147: 293-300. Clin North Am 1986; 24: 209-27. $W$. Magnetic resonance imaging of knee hyaline cartilage 